\section{A) Check for updates}

Cite this: Polym. Chem., 2018, 9, 611

\title{
The effect of linker length on ConA and DC-SIGN binding of $S$-glucosyl functionalized poly(2-oxazoline)s $\uparrow$
}

\author{
Gokhan Yilmaz, ${ }^{\mathrm{a}, \mathrm{b}, \mathrm{c}}$ Veselina Uzunova, ${ }^{\mathrm{d}}$ Manuel Hartweg, ${ }^{\mathrm{c}}$ Valentin Beyer, ${ }^{\mathrm{c}}$ \\ Richard Napier ${ }^{d}$ and C. Remzi Becer (D) *c
}

\begin{abstract}
A new monomer, 2-[2-((2,3,4,6-tetra-O-acetyl- $\beta$-D-glucopyranosylthio)propyl)]-2-oxazoline ( $\mathrm{Ac}_{4} \mathrm{Glc}-\mathrm{S}$ $\mathrm{Ox}$ ), was synthesized by direct addition of 2,3,4,6-tetra-O-acetyl-1-thio- $\beta$-D-glucopyranose ( $\mathrm{Ac}_{4} \mathrm{Glc}$-SH) to 2-isopropenyl-2-oxazoline (iPOx) in the presence of solid butyl amine resin via a thiol-ene click reaction. The living cationic ring-opening polymerization was performed to prepare copolymers of $\mathrm{Ac}_{4} \mathrm{Glc}-\mathrm{S}$ Ox with 2-ethyl-2-oxazoline (EtOx). In order to systematically investigate the effect of the S-glucosyl substituent linked to the polymer backbone with different linkers on the cloud point and the binding ability, another series of glycopolymers was prepared by a post polymerization modification method. Copolymers of 2-decenyl-2-oxazoline and 2-butenyl-2-oxazoline with EtOx were first polymerized and then reacted with $\mathrm{Ac}_{4} \mathrm{Glc}-\mathrm{SH}$. The obtained glycopolymers exhibited lower critical solution temperature behavior that could be tuned easily by manipulating the alkyl linker length. Moreover, the binding results obtained using both turbidimetry and surface plasmon resonance techniques suggested that the relationship of the linker with the polymer backbone has a critical influence on glycopolymer-lectin binding behaviour.
\end{abstract}

Received 19th November 2017 Accepted 27th December 2017 DOI: 10.1039/c7py01939d rsc.li/polymers phobic stacking. To provide a multivalent carbohydrate-lectin interaction it is very important to design different types of biological and biomedical applications, such as, pathogen detection, toxin inhibition and lectin-based biosensing. Glycopolymers (GPs) that are essentially synthetic carbohydrate containing macromolecules can display similar structural and functional features to oligosaccharides such as variations in anomeric status, linkage positions, branching, and introduction of site specific substitutions. ${ }^{9-14}$ Therefore, even though there have been great developments in the synthesis of welldefined glyco-polymers over the last decade, it is still challenging to provide precision on the control of chain lengths, monomer repeat sequences, compositions, and architectures ${ }^{15,16}$ Furthermore, in order to promote the development of glyconanoparticles for different bio-related applications such as drug delivery, biomaterials, and biotechnologies it is necessary to understand the basis of selectivity and strength in the specific interactions between glycomaterials and lectins in more detail. ${ }^{17-22}$

In general, there are two advanced synthesis strategies to prepare glycopolymers: either the direct polymerization of carbohydrate-bearing monomers or the synthesis of polymeric backbone bearing pendant reactive sites subsequently functionalized by carbohydrate moieties by using a combination of controlled/living polymerization techniques and click chemistry. ${ }^{23}$ Cationic ring opening polymerization (CROP) is one of the earliest developed living polymerization techniques, but it

\footnotetext{
${ }^{a}$ Department of Chemistry, University of Warwick, CV4 7AL, Coventry, UK
${ }^{b}$ Department of Basic Sciences, Turkish Military Academy, Ankara, Turkey

${ }^{b}$ Department of Chemistry, University of Warwick, CV4 7AL, Coventry, UK
${ }^{b}$ epartment of Basic Sciences, Turkish Military Academy, Ankara, Turkey

${ }^{c}$ Polymer Chemistry Laboratory, School of Engineering and Materials Science,

Queen Mary, University of London, E1 4NS, London, UK.

E-mail:R.Becer@QMUL.AC.UK

${ }^{d}$ Life Sciences, University of Warwick, CV4 7AL, Coventry, UK

$\dagger$ Electronic supplementary information (ESI) available. See DOI: 10.1039/ c7py01939d
} 
has not been studied extensively yet for the preparation of glycopolymers due to the limited knowledge on direct polymerization of carbohydrate-containing cyclic monomers. $^{24,25}$ Therefore, it is necessary to develop new strategies to prepare different types of glycopolymers with tunable properties via CROP. 2-Alkyl-2-oxazolines are commonly used for CROP and they are promising candidates for biological applications due to their biocompatibility, low toxicity and incorporation with different functionalities. ${ }^{26,27}$

The first study dealing with the CROP of a sugar-functionalized 2-oxazoline was reported in 2010 by Kojima et al. ${ }^{28}$ Two different $S$-galactosyl substituted oxazolines were prepared in a three-step reaction and polymerized via CROP. ${ }^{24}$ After the deprotection, the obtained poly(2-oxazoline)s with pendant sugar moieties showed specific interaction with $\mathrm{RCA}_{120}$ (Ricinus communis agglutinin). Shortly after, Schubert and coworkers reported the synthesis of a glucose-substituted 2-oxazoline monomer and its co-polymerization by CROP in the presence of 2-oxazoline-based monomers. The glucose-substituted 2-oxazoline monomer was prepared by using copper-catalyzed azide-alkyne cycloaddition (CuAAC) click chemistry after a multi-step reaction. Moreover, Schlaad and collaborators performed a post polymerization modification of a well-defined poly(2-butenyl-2-oxazoline) with a 2,3,4,6-tetra-O-acetyl-1-thio$\beta$-D-glucopyranose $\left(\mathrm{Ac}_{4} \mathrm{Glc}-\mathrm{SH}\right)$ to yield protected glycopolymers. ${ }^{29}$ Following this report, poly(2-decenyl-2-oxazoline) was clicked with an acetylated thioglucose by the Schubert group to prepare a small library of glycopolymers. ${ }^{30}$

Herein, we describe the synthesis and CROP of 2-[2$((2,3,4,6$-tetra- $O$-acetyl- $\beta$-D-glucopyranosylthio)propyl)]-2-oxazoline $\left(\mathrm{Ac}_{4} \mathrm{Glc}-S\right.$-Ox $)$ glycomonomers for the first time. To the best of our knowledge, this is the first report on one-pot preparation of 2-oxazoline based glycomonomers that does not require further purification and provide a much simplified methodology. The thiol-ene "click" reaction was performed to prepare $\mathrm{Ac}_{4} \mathrm{Glc}-\mathrm{S}$-Ox by the addition of $\mathrm{Ac}_{4} \mathrm{Glc}-\mathrm{SH}$ to 2-isopropenyl-2-oxazoline (iPOx) with a 1 to 1 molar ratio in the presence of solid butyl amine resin under a $365 \mathrm{~nm}$ UV lamp overnight. ${ }^{31}$ Due to the importance of the linker length in lectin recognition and binding kinetics, a series of GPs with different linker lengths was prepared by post polymerization modification of pendant alkene functionalities with thiols via photoaddition. In this way, copolymers of 2-decenyl-2-oxazoline (DecenOx) and 2-butenyl-2-oxazoline (ButenOx) with 2-ethyl-2-oxazoline (EtOx) were firstly prepared and then modified with $\mathrm{Ac}_{4} \mathrm{Glc}-\mathrm{SH}$ to yield the respective glycopolymers. The lower critical solution temperature (LCST) behaviour of the obtained GPs was determined to measure cloud points (CPs). Furthermore, bioactivity and bioavailability of the synthesized GPs were investigated by measuring interactions with Concanavalin A (ConA) as well as dendritic cell-specific intercellular adhesion molecule-3-grabbing non-integrin (DC-SIGN) via turbidimetry assays and SPR, respectively. ${ }^{32,33}$ The results indicated that the interaction between GPs and lectins can be enhanced dramatically by longer linkers between sugar moieties and polymer backbones.

\section{Results and discussion}

\section{Synthesis of the $S$-glucosyl substituted 2-oxazoline glycomonomer ( $\mathrm{Ac}_{4} \mathrm{Glc}-\mathrm{S}$-Ox)}

Thiol-ene click chemistry is one of the most popular click reactions since thiol and alkene components can be incorporated into a wide range of substituents. ${ }^{34}$ As depicted in Scheme 1, the thiol groups of $\mathrm{Ac}_{4} \mathrm{Glc}-\mathrm{SH}$ were reacted with the double bonds of 2-isopropenyl-2-oxazoline (iPOx) in the presence of solid-phase butyl amine resin as a catalyst in dry acetonitrile using a $365 \mathrm{~nm}$ UV lamp overnight. The thiol-ene click reaction was monitored and quantitative conversions were confirmed by ${ }^{1} \mathrm{H}-\mathrm{NMR},{ }^{13} \mathrm{C}-\mathrm{NMR}$ and ESI-MS. As seen in ${ }^{1} \mathrm{H}$ and ${ }^{13} \mathrm{C}$ NMR in Fig. 1, the glycomonomer structure was confirmed by the disappearance of the vinyl peaks at 5.5 and $5.7 \mathrm{ppm}$ of iPOx, the appearance of new peaks at 2.6-2.8 and 3.0-3.1 ppm, and also the chemical shift of the $-\mathrm{CH}_{3}$ protons of iPOx after the reaction due to difference in chemical environment indicating the formation of the corresponding thioether structure.

In the ESI-MS spectra (ESI), there is a clear peak at 475.16 $\mathrm{m} / \mathrm{z}$ that corresponds to the molecular weight of the new $S$-glucosyl substituted 2-oxazoline glycomonomer with a theoretical mass of $475.15 \mathrm{~m} / \mathrm{z}$. The product was obtained as a pale white compound with a yield of $97 \%$. Hence, there was no need to proceed with any rigorous and extensive purification, which usually leads to a loss of product in the previous synthetic methods, and thus here a simple, one-pot, efficient and robust synthetic strategy was achieved.

\section{Synthesis of ButenOx and DecenOx monomers}

2-Butenyl-2-oxazoline (ButenOx) and 2-decenyl-2-oxazoline (DecenOx) monomers were prepared in a two-step procedure as previously reported by Kempe and co-workers. Both GC-MS measurements and ${ }^{1} \mathrm{H}$-NMR spectroscopy measurements confirmed partial formation of the desired 2-oxazoline after the first step and, therefore, the second reaction was carried out without any further purification of the intermediate product. Ring closure was achieved with $\mathrm{KOH}$ in dry $\mathrm{MeOH}$ at $70^{\circ} \mathrm{C}$. The pure products were obtained after vacuum distillation with yields of $42 \%$ and $72 \%$ for ButenOx and DecenOx, respectively.

\section{Microwave-assisted copolymerization}

CROP can be affected significantly by the type of solvent, monomer concentration and temperature. ${ }^{25,35}$ Therefore, a detailed optimization study was undertaken to identify the ideal polymerization conditions. Briefly, when the polymerization reaction was carried out at $140{ }^{\circ} \mathrm{C}$, or at higher monomer concentrations, the resulting polymer exhibited a relatively high dispersity, which was also evident in the GPC trace as a shoulder in the lower elution volume region. The possible reason behind this could be the degradation of the sugar moiety or some coupling reactions between polymer chains. In the case of lower polymerization temperatures, the conversion of $\mathrm{Ac}_{4} \mathrm{Glc}-\mathrm{S}$-Ox remained low. The optimal temperature and monomer concentration were determined to be $120{ }^{\circ} \mathrm{C}$ and $1 \mathrm{M}$, respectively for further copolymerizations. 


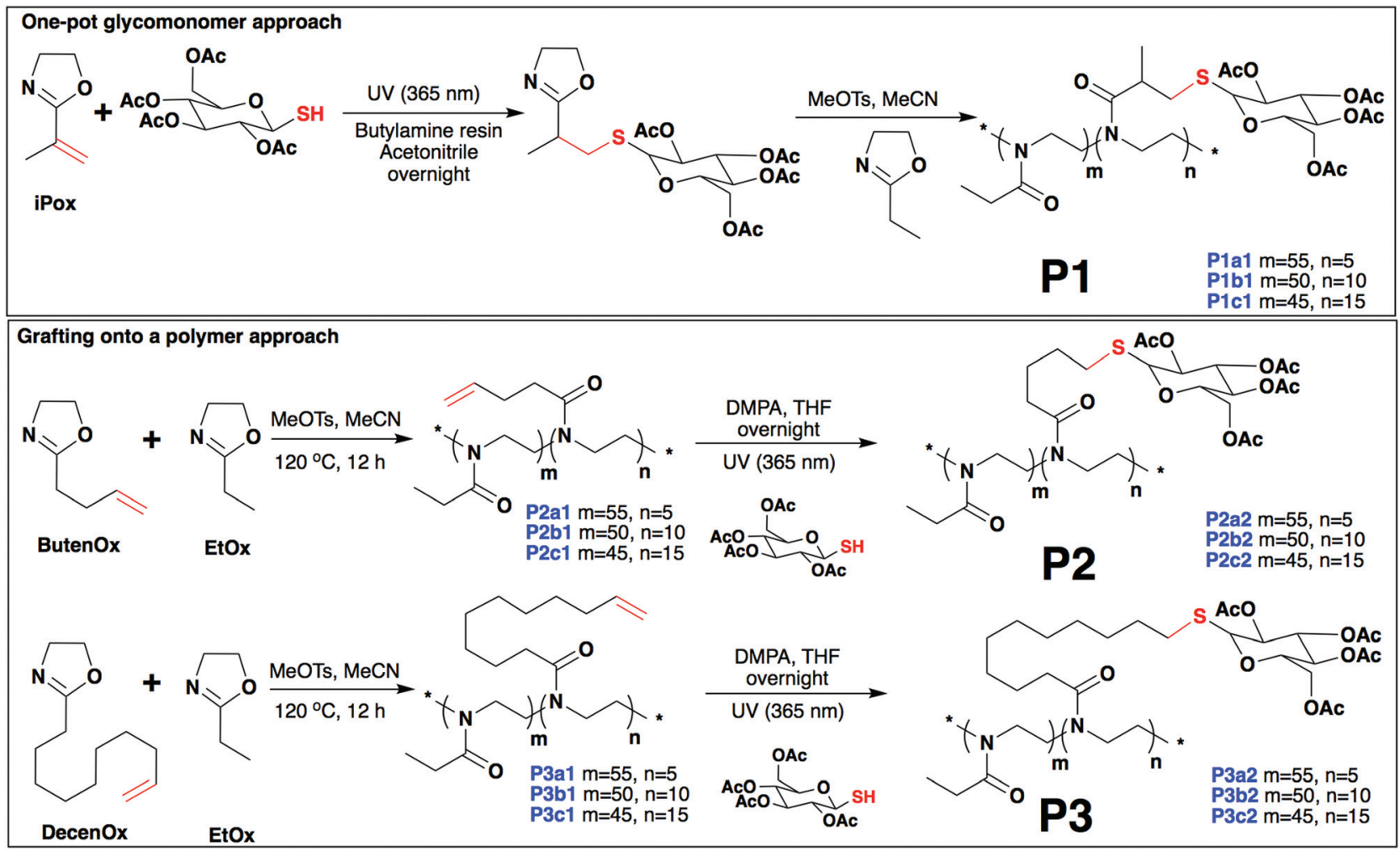

Scheme 1 Schematic representation of the synthesis of one-pot glycomonomer approach (top) and copolymerization of 2-alkenyl-2-oxazolines with 2-ethyl-2-oxazoline at various ratios and followed by the glycosylation reaction (bottom).

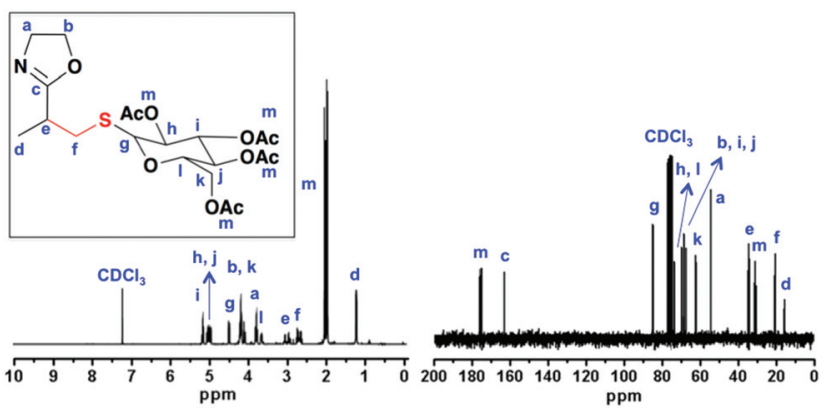

Fig. $1{ }^{1} \mathrm{H}-\mathrm{NMR}$ (left) and ${ }^{13} \mathrm{C}-\mathrm{NMR}$ (right) spectra of the $\mathrm{Ac}_{4} \mathrm{Glc}-\mathrm{S}-\mathrm{Ox}$ monomer

In order to obtain a more detailed understanding, a kinetic study of the copolymerization of EtOx and $\mathrm{Ac}_{4} \mathrm{Glc}-\mathrm{S}$-Ox was performed with a DP of 60 (EtOx 50, $\mathrm{Ac}_{4} \mathrm{Glc}-S$-Ox 10 ) and $1 \mathrm{M}$ monomer concentration at $120{ }^{\circ} \mathrm{C}$ for $12 \mathrm{~h}$ in a microwave reactor. The polymerization conversion of EtOx was monitored using GC, but the sugar substituted 2-oxazoline conversion could not be monitored by GC or ${ }^{1} \mathrm{H}-\mathrm{NMR}$ because of overlapping signals from sugars with the oxazoline ring signals at 3.8 and $4.2 \mathrm{ppm}$. Therefore, the conversion of $\mathrm{Ac}_{4} \mathrm{Glc}-S$-Ox was calculated from the GPC analysis. As depicted in Fig. 2, semilogarithmic kinetic plots display a linear increase of conversion with time for both monomers, as expected, showing the absence of termination reactions. Copolymerization of EtOx and $\mathrm{Ac}_{4} \mathrm{Glc}-\mathrm{S}$-Ox has been achieved with a relatively good control after $12 \mathrm{~h}$ according to NMR and GPC analysis.

The polymerization of EtOx proceeded significantly faster than that of $\mathrm{Ac}_{4} \mathrm{Glc}-\mathrm{S}$-Ox. This difference in reactivity of the monomers provided the obtained gradient copolymers. The number average molar mass $\left(M_{\mathrm{n}, \mathrm{GPC}}\right)$ measured in DMF by GPC increased linearly at higher monomer conversions. As shown in Table 1, the $M_{\mathrm{n}, \mathrm{GPC}}$ is found to be higher than the theoretical molar mass ( $\left.M_{\mathrm{n}, \text { Theo }}\right)$ mainly due to the difference in the chemical structure of copolymers in comparison with PS calibration standards, causing a significant difference in the hydrodynamic volume in DMF. Nevertheless, the molar mass distribution $(D)$ was kept below 1.24 throughout the entire polymerization process. Thus, the living cationic ring opening polymerization (CROP) process allowed preparation of a series of well-defined GPs (P1a2-P1c2; Table 1) by using EtOx and $\mathrm{Ac}_{4}$ Glc-S-Ox monomers at $55: 5$ (P1a1), $50: 10$ (P1b1), and $45: 15$ (P1c1) ratios. The copolymerizations of DecenOx and ButenOx with EtOx have also been achieved with a good control over the polymerization.

A more complex copolymer, P4a1, containing all monomers EtOx, ButenOx, DecenOx and $\mathrm{Ac}_{4}$ Glc-S-Ox was synthesized to compare its LCST behaviour and lectin binding ability with the other obtained GPs. The total degree of polymerization was kept as 60 with the same amount of ButenOx, DecenOx and $\mathrm{Ac}_{4} \mathrm{Glc}$-S-Ox $(8.3 \mathrm{~mol} \%$, DP $=5$ for each). All the monomers were mixed in the presence of methyl tosylate (MeOTs) as 

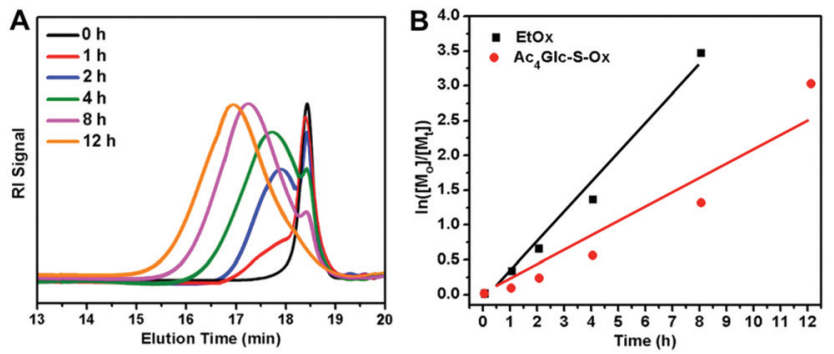

Table 1 Molar mass and polydispersity data of the deprotected glycocopolymers

\begin{tabular}{lcrllll}
\hline & & & & \multicolumn{2}{c}{ Cloud point $\left({ }^{\circ} \mathrm{C}\right)$} \\
\cline { 5 - 6 } Code & $M_{\text {n,Theo }}(\mathrm{Da})$ & $M_{\mathrm{n}, \mathrm{GPC}}(\mathrm{Da})$ & $D$ & & Heating & Cooling \\
\hline P1a2 & 7590 & 9500 & 1.21 & 61.4 & 62.3 \\
P1b2 & 8460 & 11400 & 1.20 & 80.8 & 81.2 \\
P1c2 & 9410 & 13300 & 1.21 & n.d. & n.d. \\
P2a3 & 7670 & 9900 & 1.14 & 54.3 & 55.7 \\
P2b3 & 8650 & 11700 & 1.16 & 75.9 & 76.3 \\
P2c3 & 9640 & 13800 & 1.15 & n.d. & n.d. \\
P3a3 & 8080 & 10800 & 1.16 & 47.8 & 48.7 \\
P3b3 & 9440 & 12900 & 1.16 & 36.5 & 37.3 \\
P3c3 & 10820 & 14600 & 1.17 & 27.2 & 28.4 \\
P4a3 & 10090 & 13100 & 1.24 & 64.3 & 64.7 \\
& & & & &
\end{tabular}

an initiator and acetonitrile as a solvent and then irradiated in a microwave reactor at $120^{\circ} \mathrm{C}$. Full monomer conversion was reached after $12 \mathrm{~h}$. The $M_{\mathrm{n}, \mathrm{GPC}}$ was expectedly higher than $M_{\mathrm{n}, \text { Theo }}$ with a slight increase in the $\oslash$ value.

\section{Thiol-ene photoaddition reactions of copolymers using $\mathrm{Ac}_{4} \mathrm{Glc}-\mathrm{SH}$}

Thiol-ene click reactions of poly(EtOx-ButenOx)s, poly(EtOxDecenOx)s and poly(EtOx-ButenOx-DecenOx-Ac ${ }_{4} \mathrm{Glc}-\mathrm{S}$-Ox) were carried out to obtain (P2a2-P2c2, P3a2-P3c2, P4a2) modified with $\mathrm{Ac}_{4} \mathrm{Glc}-\mathrm{SH}$ under $\mathrm{UV}$ irradiation at ambient temperature in the presence of a UV-labile radical initiator (DMPA) overnight. $\mathrm{Ac}_{4} \mathrm{Glc}-\mathrm{SH}$ was used in slight excess (1.2 eq. with respect to the double bonds) to ensure complete conversion of the ene groups. The reaction was monitored by ${ }^{1} \mathrm{H}$ NMR spectroscopy and GPC analysis.

As shown in Fig. 3A, S3, S5 and S7, $\uparrow$ the peaks of the alkene groups of copolymers at 5.8-6.0 ppm disappeared and the acetyl protecting groups of sugars appeared at $2.0 \mathrm{ppm}$ as the thiol-ene reaction progressed. The GPC traces of copolymers also showed significant shifts due to an increase of the molar mass after the thiol-ene photoaddition of $\mathrm{Ac}_{4} \mathrm{Glc}-\mathrm{SH}$ (Fig. 4). Moreover, there was no considerable increase in $D$ values. The FT-IR spectra revealed the appearance of a stretch at $1745 \mathrm{~cm}^{-1}$, which originates from the ester bond of acetyl protecting groups (Fig. 3B, S4, S6 and S8†).

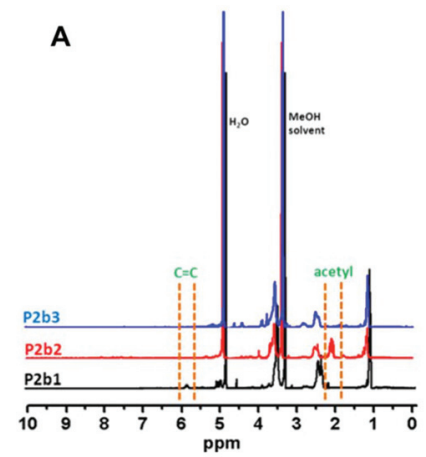

B

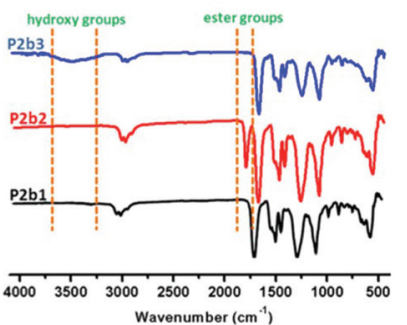

Fig. 3 (A) ${ }^{1} \mathrm{H}$ NMR characterization $\left(400 \mathrm{MHz}, \mathrm{CD}_{3} \mathrm{OD}\right)$ and (B) FT-IR spectra of the obtained copolymers P2b1, P2b2 (thiol-ene product) and P2b3 (after deacetylation).
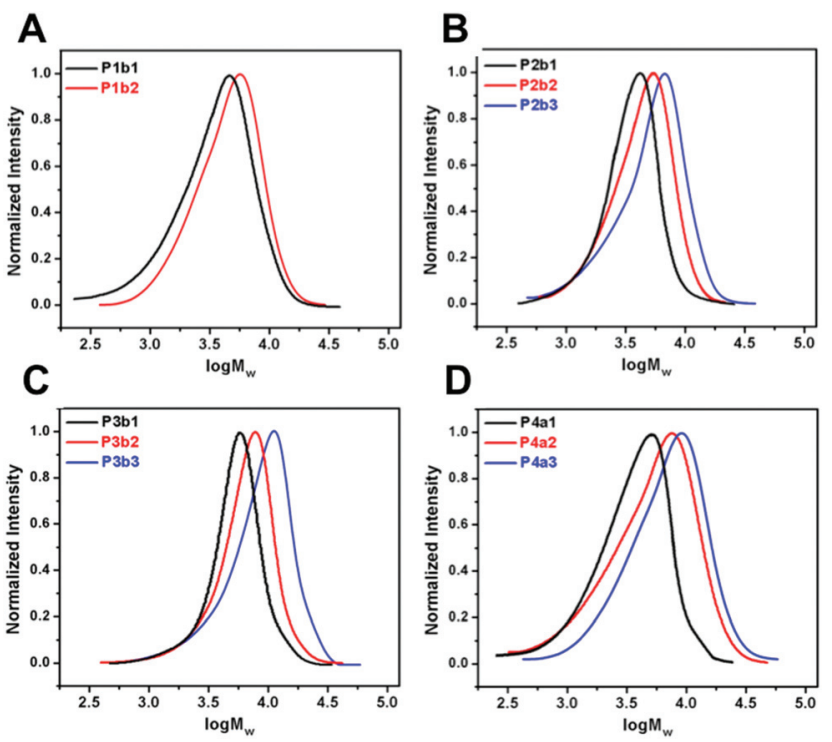

Fig. 4 GPC traces of the copolymers of each series: (A) poly(EtOx-co$\left.\mathrm{Ac}_{4} \mathrm{Glc}-\mathrm{S}-\mathrm{Ox}\right) \mathrm{s}$ before and after deprotection; (B) poly(EtOx-coButenOx)s; (C) poly(EtOx-co-DecenOx)s and (D) copoly(EtOx-ButenOxDecenOx-Ac ${ }_{4} \mathrm{Glc}-\mathrm{S}-\mathrm{Ox}$ ) before and after thiol-ene and deprotection reactions. 


\section{Deprotection of the obtained copolymers}

Deprotection of all copolymers bearing protected sugar moieties was performed in $\mathrm{MeOH}$ with sodium methoxide for $3 \mathrm{~h}$ at room temperature. Deprotection was followed by ${ }^{1} \mathrm{H}$ NMR and FT-IR. As seen in Fig. 3A, S3, S5 and S7, $\dagger$ the peak of the acetate groups at $2.0 \mathrm{ppm}$ disappeared in the ${ }^{1} \mathrm{H}$ NMR spectra, and the FT-IR signal at $1745 \mathrm{~cm}^{-1}$ disappeared. Deacetylation of the protected sugar residues produced free hydroxyl groups that caused a broad stretch at about $3350 \mathrm{~cm}^{-1}$ in the FT-IR spectrum (Fig. 3B, S4, S6 and S8 $\dagger$ ). Furthermore, DMF GPC analysis revealed the shift of elution peaks after the deprotection reaction due to a change in the hydrodynamic volume (Fig. 5). Even if the theoretical molar mass decreased after the reaction, the $M_{\mathrm{n}, \mathrm{GPC}}$ increased, as shown by clear shifts to lower elution volumes. This is probably due to increased solubility of the free hydroxyl groups of sugar moieties creating a larger hydrodynamic volume.

\section{Solubility of the glycopolymers in aqueous media}

Thermal phase transition temperatures of the GPs were measured at a polymer concentration of $5 \mathrm{mg} \mathrm{mL}^{-1}$ in the temperature range from $20^{\circ} \mathrm{C}$ and $80{ }^{\circ} \mathrm{C}$ in order to investigate CPs. In general, LCST behaviour can be influenced by several properties, such as, the chain length, architecture, hydrophilic-hydrophobic balance and also concentration of the polymers. Recent reports have highlighted the LCST behaviour of poly(oxazoline)-based GPs with different carbohydrate contents. $^{30}$

As seen in Fig. 6, the sugar content has a dramatic effect on the LCST of the GPs. For P1a2-P1c2 (deprotected poly(EtOx-co$\mathrm{Ac}_{4}$ Glc-S-Ox)s) and P2a3-P2c3 (deprotected glucose clicked poly(EtOx-co-ButenOx)s), increasing the number of sugar moi-
A
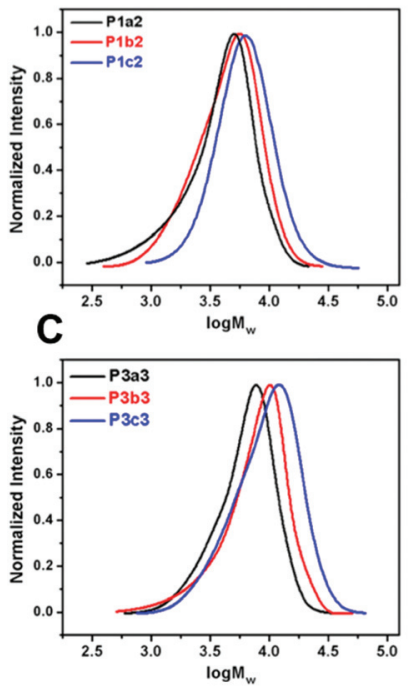

\section{B}
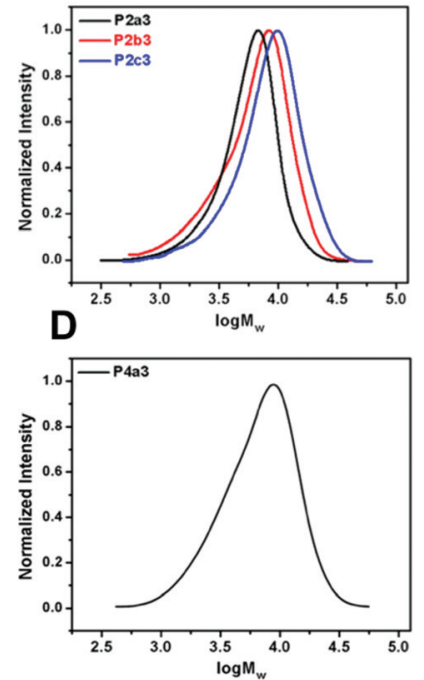

Fig. 5 GPC traces of the (deprotected) glyco-copolymers of each series: (A) poly(EtOx-CO-Ac $\left.{ }_{4} \mathrm{Glc}-\mathrm{S}-\mathrm{Ox}\right) \mathrm{s}$; (B) poly(EtOx-Co-ButenOx)s; (C) poly(EtOx-co-DecenOx)s and (D) copoly(EtOx-ButenOx-DecenOx$\left.\mathrm{Ac}_{4} \mathrm{Glc}-\mathrm{S}-\mathrm{Ox}\right)$.
A
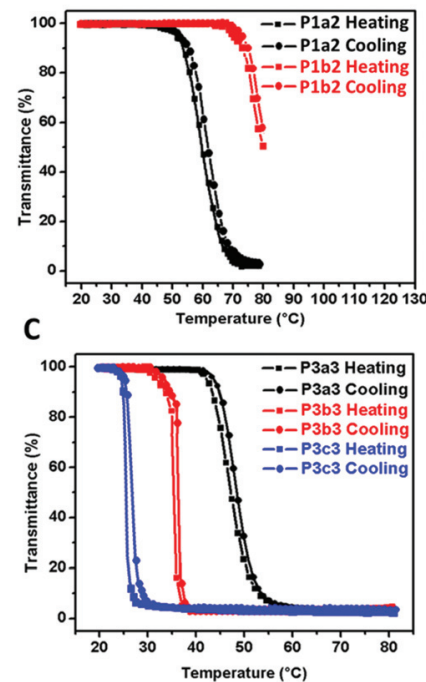

B
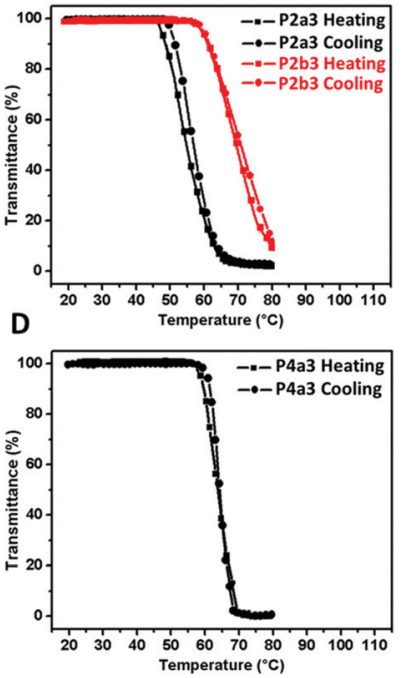

Fig. 6 Turbidity curves for the determination of the cloud points of the (deprotected) glyco-copolymers of each series: (A) poly(EtOx-Co$\left.\mathrm{Ac}_{4} \mathrm{Glc}-\mathrm{S}-\mathrm{Ox}\right) \mathrm{s} ; \quad$ (B) poly(EtOx-co-ButenOx)s; (C) poly(EtOx-coDecenOx)s and (D) copoly(EtOx-ButenOx-DecenOx-Ac ${ }_{4}$ Glc-S-Ox).

eties results in an increase in the LCST. In general, the LCST values of all GPs revealed successive phase transitions with small hysteresis. It is clear that there are considerable differences in the LCST values between the GPs with the same amount of sugar content. This shows that the spacer length between the polymer backbone and the sugar residues has a significant influence on the cloud point, probably because of the increase in hydrophobicity of the copolymers with longer alkyl chain substituents.

As seen in Table 1 and Fig. 6, the cloud points of GPs with the shortest alkyl linker (P1a2-P1c2) were found to be higher than those of the other GPs with longer alkyl chains. P1c2 including the highest degree of glucose has the highest LCST value. In contrast, the CPs of the GPs bearing decenyl chain sugar substituents (P3a3-P3c3) exhibited the lowest CPs. Furthermore, even though the fractional sugar content increased, the CP temperature of the GPs decreased (Table 1), similar to results previously presented by Schubert and co-workers. ${ }^{30}$

\section{Lectin binding studies}

Turbidimetry is the most common and straightforward technique for determining lectin binding and understanding the recognition events between a polymer and a protein. All glycopolymers were dissolved in freshly prepared HBS buffer solutions $(640 \mu \mathrm{M})$ and then added into a ConA solution $(120 \mu \mathrm{M})$ and placed in the UV spectrometer. The absorbance of the mixture was recorded at $420 \mathrm{~nm}$ for $15 \mathrm{~min}$ every $0.12 \mathrm{~s}$.

Polymer P3c3, containing the longest linker, showed the highest binding signal from all the glycopolymers (Fig. 7). The structurally more complex GP, P4a3, also provided a strong binding. Basically, the binding activity showed a correlation with sugar density. P1a2 interacted weakly with ConA, consist- 


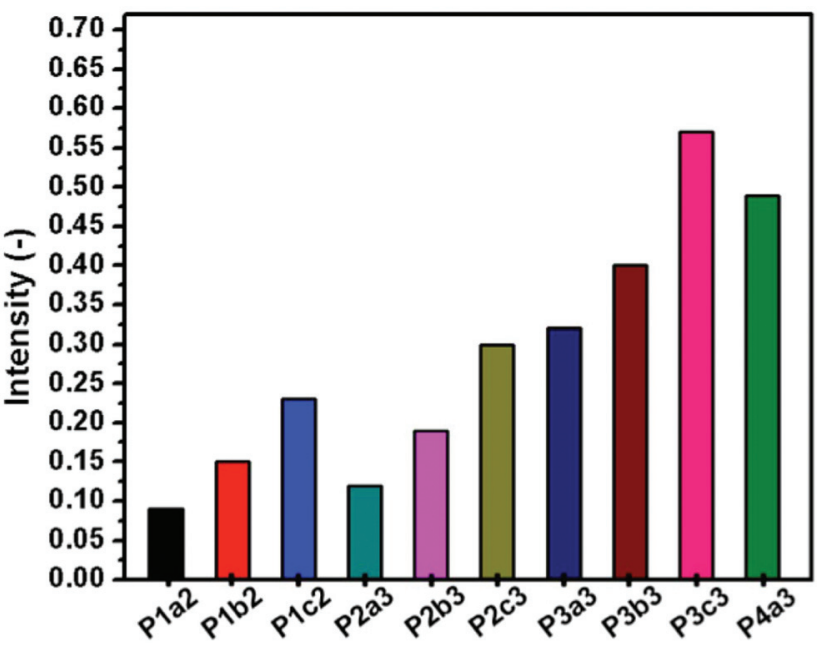

Fig. 7 Turbidity measurements of the obtained glycopolymers with ConA.

ent with its low sugar density, although this polymer also has a shorter linker, which may decrease the accessibility of sugar residues. As seen in Fig. 7 and Fig. S9, $\dagger$ there are no big differences in the binding activity between any of the GPs bearing short linkers (P1a2-P1c2), whereas the P3 series showed a significant increase in the binding ability with ConA.

To confirm the results obtained from the turbidimetry assay, more detailed analysis was carried out using SPR. The C-type dendritic cell lectin, namely DC-SIGN, was used in kinetic experiments with GPs (Fig. 8). The results of the SPR experiments are in excellent agreement with the turbidimetry assay, confirming that the binding between GPs and lectins was enhanced by longer linkers. As depicted in Fig. 8 and Fig. S10, $\uparrow$ P3c3 showed the strongest binding with DC-SIGN

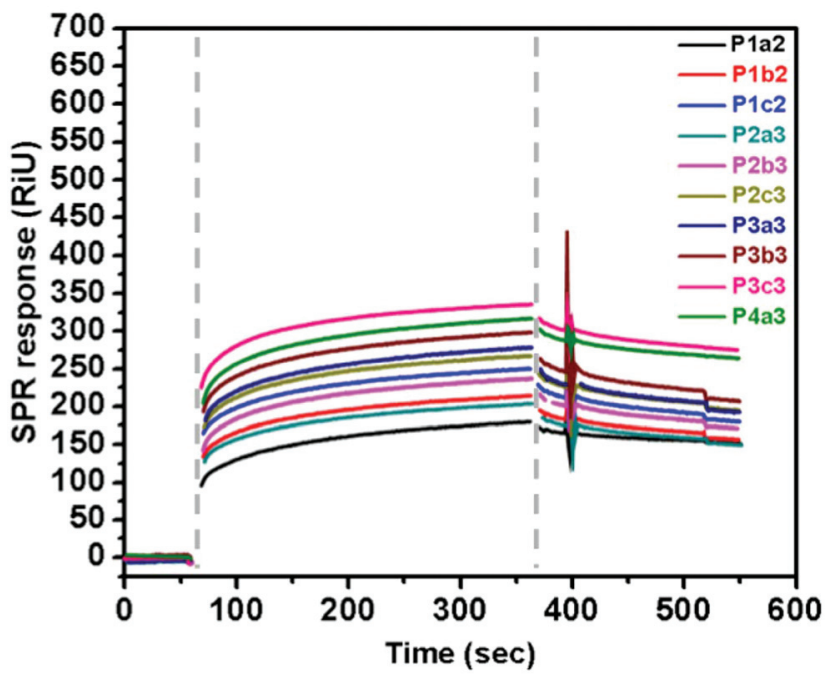

Fig. 8 Comparison of the binding of glycopolymers with DC-SIGN at a concentration of $16 \mu \mathrm{M}$. (The grid lines represent injection time of association and dissociation.)
Table 2 Kinetic binding data of glycopolymers and DC-SIGN as calculated by SPR

\begin{tabular}{lllll}
\hline Code & $k_{\mathrm{a}}(1 / \mathrm{M} \mathrm{s})$ & $k_{\mathrm{d}}(1 / \mathrm{s})$ & $K_{\mathrm{a}}(1 / \mathrm{M})$ & $R_{\max }(\mathrm{RU})$ \\
\hline P1a2 & $2.44 \times 10^{3}$ & $1.09 \times 10^{-9}$ & $2.24 \times 10^{12}$ & 180 \\
P1b2 & $1.05 \times 10^{3}$ & $4.66 \times 10^{-8}$ & $2.26 \times 10^{10}$ & 215 \\
P1c2 & $1.36 \times 10^{3}$ & $1.64 \times 10^{-7}$ & $8.28 \times 10^{9}$ & 250 \\
P2a3 & $1.10 \times 10^{3}$ & $3.90 \times 10^{-8}$ & $2.81 \times 10^{10}$ & 200 \\
P2b3 & $1.24 \times 10^{3}$ & $3.54 \times 10^{-8}$ & $3.52 \times 10^{10}$ & 200 \\
P2c3 & $1.70 \times 10^{3}$ & $5.25 \times 10^{-8}$ & $3.24 \times 10^{10}$ & 270 \\
P3a3 & $1.66 \times 10^{3}$ & $1.74 \times 10^{-8}$ & $9.52 \times 10^{10}$ & 280 \\
P3b3 & $2.03 \times 10^{3}$ & $1.67 \times 10^{-7}$ & $1.21 \times 10^{10}$ & 300 \\
P3c3 & $3.73 \times 10^{3}$ & $2.04 \times 10^{-7}$ & $1.82 \times 10^{10}$ & 335 \\
P4a3 & $3.76 \times 10^{3}$ & $4.02 \times 10^{-7}$ & $9.36 \times 10^{9}$ & 315
\end{tabular}

whereas P1a2 was the weakest. The rest of GPs demonstrated intermediate interaction profiles according to their sugar density and linker length.

According to the kinetic binding parameters of all GPs, there is no considerable difference in association rate constants $\left(k_{\mathrm{a}}\right)$, but an increase in dissociation rate constants $\left(k_{\mathrm{d}}\right)$ was observed. The binding kinetics exhibit the characteristics of multivalent interactions, with initial association rates slowing rapidly, probably due to the "zipper effect" as sugar residues along the same GP fill local lectin binding sites without adding any further mass to the chip surface. ${ }^{36}$ Dissociation rate constants are also complex to interpret and very slow dissociation rates are seen in all GP samples, which are expected in multivalent binding interactions. As one sugar dissociates, it is unable to diffuse away because it remains part of the polymer complex, promoting rebinding and very slow dissociation kinetics. Interestingly, even though P1a2 showed the weakest binding according to turbidimetry (Fig. 6), which is best indicator of complex association, it demonstrated the highest affinity because of a very low dissociation rate constant (approximately 100-fold) (Table 2). This suggests that the short linker lengths may restrict egress of sugar groups away from the lectin once the multivalent complex is made, a characteristic that may be worth further investigation.

\section{Conclusions}

In summary, a series of poly(2-oxazoline)s containing different alkyl linker lengths and sugar units on the polymer backbone were synthesized via CROP and thiol-ene click reaction. A simple, one-pot, and versatile synthetic strategy was described for the preparation of a novel $S$-glucosyl substituted 2-oxazoline glycomonomer via thiol-ene click chemistry. A systematic study on the cloud points of these polymers showed that it is possible to provide an efficient control on the thermo-responsive behaviour of the synthesized glycopolymers with the targeted LCST value. Turbidimetry and SPR results confirmed that the length of a linker between the carbohydrate and polymer backbone has a critical influence on sugar binding activity. It is likely that the increased flexibility of longer linkers provides more accessibility and promotes multivalent 
binding interactions with lectins. There is no doubt that the ability to control linkage groups combined with reliable synthesis will be advantageous for the application of the glycopolymer technology to medicine and biotechnology.

\section{Conflicts of interest}

There are no conflicts of interest to declare.

\section{Acknowledgements}

This study is supported by Turkish Army Forces and Engineering and Physical Sciences Research Council (EP/ P009018/1).

\section{References}

1 B. Le Droumaguet and J. Nicolas, Recent advances in the design of bioconjugates from controlled/living radical polymerization, Polym. Chem., 2010, 1(5), 563-598.

2 Y. Miura, Y. Hoshino and H. Seto, Glycopolymer Nanobiotechnology, Chem. Rev., 2016, 116(4), 1673-1692.

3 C. R. Becer, The Glycopolymer Code: Synthesis of Glycopolymers and Multivalent Carbohydrate-Lectin Interactions, Macromol. Rapid Commun., 2012, 33(9), 742-752.

4 L. L. Kiessling and J. C. Grim, Glycopolymer probes of signal transduction, Chem. Soc. Rev., 2013, 42(10), 44764491.

5 D. A. Mitchell, Q. Zhang, L. Voorhaar, D. M. Haddleton, S. Herath, A. S. Gleinich, H. S. Randeva, M. Crispin, H. Lehnert, R. Wallis, S. Patterson and C. R. Becer, Manipulation of cytokine secretion in human dendritic cells using glycopolymers with picomolar affinity for DC-SIGN, Chem. Sci., 2017, 8(10), 6974-6980.

6 R. J. Liu, D. Patel, H. R. C. Screen and C. R. Becer, A(2) B-Miktoarm Glycopolymer Fibers and Their Interactions with Tenocytes, Bioconjugate Chem., 2017, 28(7), 1955-1964.

7 C. Gauche and S. Lecommandoux, Versatile design of amphiphilic glycopolypeptides nanoparticles for lectin recognition, Polymer, 2016, 107, 474-484.

8 C. von der Ehe, C. Weber, M. Gottschaldt and U. S. Schubert, Immobilized glycopolymers: Synthesis, methods and applications, Prog. Polym. Sci., 2016, 57, 64102.

9 M. W. Jones, L. Otten, S. J. Richards, R. Lowery, D. J. Phillips, D. M. Haddleton and M. I. Gibson, Glycopolymers with secondary binding motifs mimic glycan branching and display bacterial lectin selectivity in addition to affinity, Chem. Sci., 2014, 5(4), 1611-1616.

10 G. Yilmaz and C. R. Becer, Precision glycopolymers and their interactions with lectins, Eur. Polym. J., 2013, 49(10), 3046-3051.

11 Y. Hoshino, M. Nakamoto and Y. Miura, Control of ProteinBinding Kinetics on Synthetic Polymer Nanoparticles by
Tuning Flexibility and Inducing Conformation Changes of Polymer Chains, J. Am. Chem. Soc., 2012, 134(37), 1520915212.

12 A. W. Du, H. Lu and M. H. Stenzel, Cationic glycopolymers through controlled polymerisation of a glucosamine-based monomer mimicking the behaviour of chitosan, Polym. Chem., 2017, 8(11), 1750-1753.

13 Q. Zhang, G. Nurumbetov, A. Simula, C. Zhu, M. Li, P. Wilson, K. Kempe, B. Yang, L. Tao and D. M. Haddleton, Synthesis of well-defined catechol polymers for surface functionalization of magnetic nanoparticles, Polym. Chem., 2016, 7(45), 7002-7010.

14 G. Yilmaz, L. Messager, A. S. Gleinich, D. A. Mitchell, G. Battaglia and C. R. Becer, Glyconanoparticles with controlled morphologies and their interactions with a dendritic cell lectin, Polym. Chem., 2016, 7(41), 6293-6296.

15 Q. Zhang, J. Collins, A. Anastasaki, R. Wallis, D. A. Mitchell, C. R. Becer and D. M. Haddleton, SequenceControlled Multi-Block Glycopolymers to Inhibit DC-SIGNgp120 Binding, Angew. Chem., Int. Ed., 2013, 52(16), 44354439.

16 J. F. Lutz, Sequence-controlled polymerizations: the next Holy Grail in polymer science?, Polym. Chem., 2010, 1(1), 55-62.

17 A. Dag, H. Lu and M. Stenzel, Controlling the morphology of glyco-nanoparticles in water using block copolymer mixtures: the effect on cellular uptake, Polym. Chem., 2015, 6(45), 7812-7820.

18 N. Cakir, G. Hizal and C. R. Becer, Supramolecular glycopolymers with thermo-responsive self-assembly and lectin binding, Polym. Chem., 2015, 6(37), 6623-6631.

19 X. Li and G. Chen, Glycopolymer-based nanoparticles: synthesis and application, Polym. Chem., 2015, 6(9), 14171430.

20 Q. Zhang, A. Anastasaki, G.-Z. Li, A. J. Haddleton, P. Wilson and D. M. Haddleton, Multiblock sequence-controlled glycopolymers via $\mathrm{Cu}(0)$-LRP following efficient thiolhalogen, thiol-epoxy and CuAAC reactions, Polym. Chem., 2014, 5(12), 3876-3883.

21 I. Kurtulus, G. Yilmaz, M. Ucuncu, M. Emrullahoglu, C. R. Becer and V. Bulmus, A new proton sponge polymer synthesized by RAFT polymerization for intracellular delivery of biotherapeutics, Polym. Chem., 2014, 5(5), 1593-1604.

22 Y. Zhao, Y. Zhang, C. Wang, G. Chen and M. Jiang, Role of Protecting Groups in Synthesis and Self-Assembly of Glycopolymers, Biomacromolecules, 2017, 18(2), 568-575.

23 S. Slavin, J. Burns, D. M. Haddleton and C. R. Becer, Synthesis of glycopolymers via click reactions, Eur. Polym. J., 2011, 47(4), 435-446.

24 K. Kempe, C. Weber, K. Babiuch, M. Gottschaldt, R. Hoogenboom and U. S. Schubert, Responsive Glyco-poly (2-oxazoline)s: Synthesis, Cloud Point Tuning, and Lectin Binding, Biomacromolecules, 2011, 12(7), 2591-2600.

25 K. Kempe, C. R. Becer and U. S. Schubert, MicrowaveAssisted Polymerizations: Recent Status and Future Perspectives, Macromolecules, 2011, 44(15), 5825-5842. 
26 P. Wilson, P. C. Ke, T. P. Davis and K. Kempe, Poly(2-oxazoline)-based micro- and nanoparticles: A review, Eur. Polym. J., 2017, 88, 486-515.

27 K. Kempe, S. L. Ng, K. F. Noi, M. Mullner, S. T. Gunawan and F. Caruso, Clickable Poly(2-oxazoline) Architectures for the Fabrication of Low-Fouling Polymer Capsules, ACS Macro Lett., 2013, 2(12), 1069-1072.

28 A. Takasu and H. Kojima, Synthesis and ring-opening polymerizations of novel S-glycooxazolines, J. Polym. Sci., Part A: Polym. Chem., 2010, 48(24), 5953-5960.

29 A. Gress, A. Völkel and H. Schlaad, Thio-Click Modification of Poly[2-(3-butenyl)-2-oxazoline], Macromolecules, 2007, 40(22), 7928-7933.

30 K. Kempe, T. Neuwirth, J. Czaplewska, M. Gottschaldt, R. Hoogenboom and U. S. Schubert, Poly(2-oxazoline) glycopolymers with tunable LCST behavior, Polym. Chem., 2011, 2(8), 1737-1743.

31 M. A. Cortez and S. M. Grayson, Thiol-Ene Click Functionalization and Subsequent Polymerization of 2-Oxazoline Monomers, Macromolecules, 2010, 43(9), 4081-4090.
32 Q. Zhang, L. Su, J. Collins, G. S. Chen, R. Wallis, D. A. Mitchell, D. M. Haddleton and C. R. Becer, Dendritic Cell Lectin-Targeting Sentinel-like Unimolecular Glycoconjugates To Release an Anti-HIV Drug, J. Am. Chem. Soc., 2014, 136(11), 4325-4332.

33 Y. Z. Gou, J. Geng, S. J. Richards, J. Burns, C. R. Becer and D. M. Haddleton, A detailed study on understanding glycopolymer library and Con A interactions, J. Polym. Sci., Part A: Polym. Chem., 2013, 51(12), 2588-2597.

34 A. B. Lowe, Thiol-ene "click" reactions and recent applications in polymer and materials synthesis: a first update, Polym. Chem., 2014, 5(17), 4820-4870.

35 R. M. Paulus, C. R. Becer, R. Hoogenboorn and U. S. Schubert, Acetyl halide initiator screening for the cationic ring-opening polymerization of 2-ethyl-2-oxazoline, Macromol. Chem. Phys., 2008, 209(8), 794-800.

36 N. C. Norris, R. J. Bingham, G. Harris, A. Speakman, R. P. O. Jones, A. Leech, J. P. Turkenburg and J. R. Potts, Structural and Functional Analysis of the Tandem $\beta$-Zipper Interaction of a Streptococcal Protein with Human Fibronectin, J. Biol. Chem., 2011, 286(44), 38311-38320. 